\title{
THERMODYNAMICS OF PARACRYSTALLINE SILICON
}

P. M. Voyles, Department of Physics, University of Illinois at UrbanaChampaign, 1110 West Green Street, Urbana, IL 61801

M. M. J. Treacy, NEC Research Institute, 4 Independence Way, Princeton, NJ 08540

J. Murray Gibson, Materials Science Division, Argonne National Laboratory, 9700 South Cass Avenue, Argonne, IL 60439-4838

April 2000

The submitted manuscript has
been created by the University of
Chicago as Operator of Argonne
National Laboratory ("Argonne")
under Contract No. W-31-109-
ENG-38 with the U.S. Department
of Energy. The U.S. Govemment
retains for itself, and others acting
on its behalf, a paid-up, non
exclusive, irrevocable worldwide
license in said article to reproduce,
prepare derivative works,
distribute copies to the public, and
perform publicly and display
publicly, by or on behalf of the
Government.

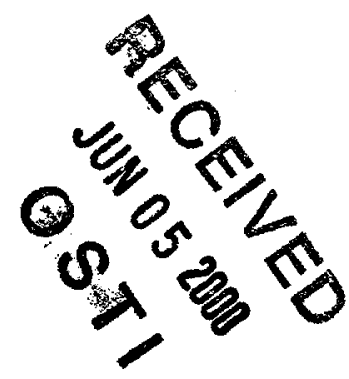

Distribution:

1-2. PRS

3. J. M. Gibson

4. L. E. Rehn

5. J. Coble

6. File

Conference Paper to be presented at the 2000 Spring Meeting of the Materials Research Society, San Francisco, CA, April 24-28, 2000.

*Work supported by the U.S. Department of Energy, Office of Science under contract No. W-31-109-Eng-38. 


\section{DISCLAIMER}

This report was prepared as an account of work sponsored by an agency of the United States Government. Neither the United States Government nor any agency thereof, nor any of their employees, make any warranty, express or implied, or assumes any legal liability or responsibility for the accuracy, completeness, or usefulness of any information, apparatus, product, or process disclosed, or represents that its use would not infringe privately owned rights. Reference herein to any specific commercial product, process, or service by trade name, trademark, manufacturer, or otherwise does not necessarily constitute or imply its endorsement, recommendation, or favoring by the United States Government or any agency thereof. The views and opinions of authors expressed herein do not necessarily state or reflect those of the United States Government or any agency thereof. 


\section{DISCLAIMER}

Portions of this document may be illegible in electronic image products. Images are produced from the best available original document. 


\title{
Thermodynamics of Paracrystalline Silicon
}

\author{
P. M. Voyles, ${ }^{1,2}$ M. M. J. Treacy, ${ }^{2}$ and J. M. Gibson ${ }^{3}$
}

${ }^{1}$ Dept. of Physics, Univ. of Illinois at Urbana-Champaign, 1110 W. Green St., Urbana, IL 61801

${ }^{2}$ NEC Research Institute, 4 Independence Way, Princeton, NJ 08540

${ }^{3}$ Materials Science Division, Argonne National Laboratory, 9700 Cass Ave., Argonne, IL 60439

\begin{abstract}
Fluctuation microscopy experiments have shown that the as-deposited structure of amorphous silicon thin films is paracrystalline. A paracrystal consists of small $(<3 \mathrm{~nm}$ in diameter) topologically crystalline grains separated by a disordered matrix. Here we consider the thermodynamics of paracrystalline silicon as a function of the grain size and the temperature. We offer a simple model that qualitatively explains the observed metastability of the ordered structure at low temperature $(300 \mathrm{~K})$, the relaxation towards a more disordered structure at intermediate temperatures $(600 \mathrm{~K})$, and the recrystallization at high temperatures $(1000 \mathrm{~K})$.
\end{abstract}

\section{INTRODUCTION}

It has been generally believed that the structure of tetrahedral amorphous semiconductors is a continuous random network (CRN). A CRN is a four-connected network in which rotations of the dihedral angle introduce long-range disorder. CRNs generated by atomistic computer simulation have successfully modeled the physical properties of amorphous semiconductors [1]. The CRN model has also been the primary interpretive framework for previous work on the thermodynamics and phase transitions of amorphous semiconductors $[2,3,4,5]$.

We have introduced a different model for the as-deposited structure of amorphous semiconductor thin films called the paracrystalline (PC) model. This model was motivated by experimental results from fluctuation microscopy [6], a transmission electron microscopy technique that is particularly sensitive to medium-range order (MRO). MRO is any structure at length scales between the short-range order and long-range disorder amorphous semiconductors are known to have from x-ray diffraction studies [7]. Using fluctuation microscopy, we have shown that thin films of amorphous silicon (a-Si) and amorphous germanium (a-Ge) show more MRO than is present in a CRN [8] and that this $\mathrm{MRO}$ is reduced by intermediate temperature $(600 \mathrm{~K})$ thermal annealing [9].

Fluctuation microscopy simulations from paracrystalline molecular dynamics (MD) model structures show the greater degree of MRO observed in real materials [8]. A PC structure is comprised of small $(<3 \mathrm{~nm}$ diameter) grains which have topologically crystalline bonding [10] but are strongly strained. These grains may be embedded in a more disordered matrix. The strained grains do not contribute visibly to the diffraction signal of the material but do provide subtle MRO that can be detected by fluctuation microscopy.

Here paper we explore the thermodynamics of paracrystalline silicon (pc-Si). In particular, we know that the material is PC in its as-deposited state $(300 \mathrm{~K})$, that intermediate-temperature $(600 \mathrm{~K})$ annealing drives it towards greater disorder, and that high-temperature $(1000 \mathrm{~K})$ annealing drives it towards greater order (crystallization). In the next section we describe a simple thermodynamic model of a compact of PC grains whose free energy landscape provides pathways for both these transformations. This model also provides an alternative explanation for 
previous differential scanning calorimetry (DSC) results on a-Si and a-Ge $[2,3,4]$. In the third section we consider the rates at which these transitions occur as a function of temperature.

\section{SIMPLE THERMODYNAMIC MODEL}

We consider the free energy of a PC compact as a function of the average grain size. In the limit of large grain size, this model becomes polycrystalline silicon. In the small-grain limit the model becomes a CRN. Our previous model [8] did not have the correct small-grain limit.

The model of a PC compact consists of PC grains separated by a skin of more disordered $\mathrm{CRN}$-like material. MD simulations of high-energy twist grain boundaries in $\mathrm{Si}$ have found such a skin [11]. For simplicity, we assume grains of uniform size. The geometry of the system is defined by the volume and surface area of the grains and the volume of the disordered matrix. The volume of each grain $v=K_{1} r^{3}$, and the surface area of the grains $S=K_{2} r^{2}$, where $r$ is a characteristic radius of the particle and $K_{1}$ and $K_{2}$ are constants determined by the grain's shape. The volume of the disordered matrix associated with one grain $\Delta=K_{1}\left[(r+d)^{3}-r^{3}\right]$, where $d$ is the half-width of the skin between the grains.

There is a free energy associated with each geometric factor. $\mu_{c}$ is the volume free energy of the crystalline grains, $\mu_{d}$ is the volume free energy of the disordered phase, and $\sigma$ is the excess free energy of interface between the crystalline phase and the disordered phase. We choose the liquid as the reference level, so the volume free energies are negative.

There is also a free energy contribution associated with the stress induced by the difference in density between the two phases. Amorphous silicon is $10 \%$ less dense than crystalline silicon [12]. This leads to a strain energy that can be approximated by

$$
\Delta G_{s}=c Y \frac{\varepsilon_{0}^{2}}{(1-v)^{2}} \frac{r d}{(r+d)^{2}}=\mu_{s} \frac{r d}{(r+d)^{2}},
$$

where $Y$ is the Young's modulus and $v$ is the Poisson's ratio of the crystalline phase, $\varepsilon_{0}$ is the rms strain, and $c$ is a constant associated with the geometry and dimensionality of the system. $\mu_{s}$ is the derived coefficient of the strain energy per unit volume.

Combining all these terms, we can write the free energy difference from the liquid per unit volume of a PC compact $\Delta G$ as

$$
\begin{aligned}
\Delta G & =\frac{1}{(r+d)^{3}}\left\{K_{1} r^{3} \mu_{c}+K_{1}\left[(r+d)^{3}-r^{3}\right] \mu_{d}+K_{2} r^{2} \sigma\right\}+\mu_{s} \frac{r d}{(r+d)^{2}}, \\
& =\frac{r^{3}}{(r+d)^{3}}\left(\mu_{c}-\mu_{d}+\frac{K \sigma}{r}\right)+\mu_{s} \frac{r d}{(r+d)^{2}}+\mu_{d}
\end{aligned}
$$

where $K \equiv K_{2} / K_{1}$, a constant describing the surface to volume ratio of the shape of the grains.

The model as expressed in Eq. (2) has a number of useful properties. First, we see that the physically meaningful parameters are $\sigma,\left(\mu_{c}-\mu_{d}\right)$ (which is negative, since the crystal is the lowest energy phase), $\mu_{s}$, and $K$. Eq. (2) also has the appropriate limits. As $r \rightarrow \infty$, the compact 
Table I: Numerical values for thermodynamics materials constants.

\begin{tabular}{c|c|c}
\hline \hline Parameter & Value & Ref. \\
\hline$\mu_{c}$ & $-50.5 \mathrm{~kJ} / \mathrm{mol}$ & 2 \\
$\mu_{d}$ & $-38.8 \mathrm{~kJ} / \mathrm{mol}$ & 3 \\
$\mu_{s}$ & $0.62 \mathrm{~kJ} / \mathrm{mol}$ & 1 \\
$\sigma$ & $0.31 \mathrm{~J} / \mathrm{m}^{2}$ & 13 \\
$\delta$ & $7 \AA$ & 11 \\
\hline \hline
\end{tabular}

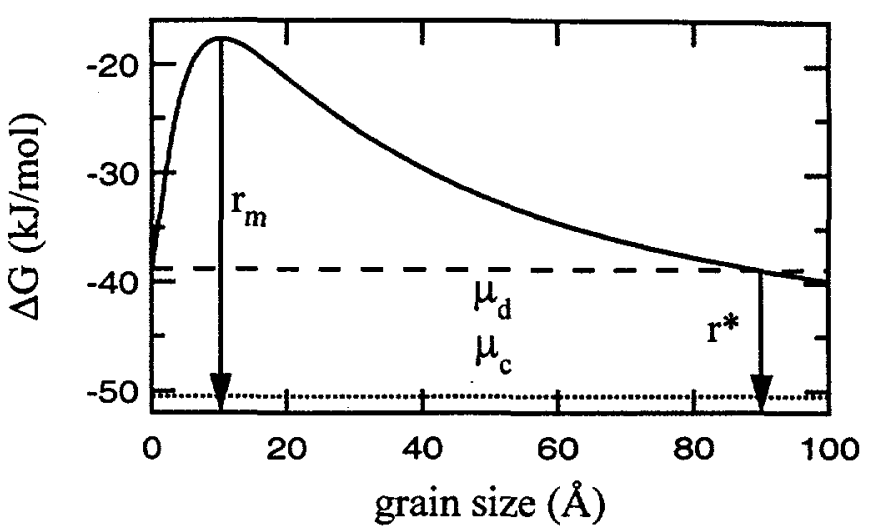

Figure 1: $\Delta G$ for a simple cubic lattice of cubic grains as a function of grain size.

becomes a polycrystalline, and $\Delta G \rightarrow \mu_{c}$. As $r \rightarrow 0$, the compact becomes only the disordered phase, and $\Delta G \rightarrow \mu_{d}$. Most importantly, Eq. (2) has a maximum at $r_{m}$ given by

$$
r_{m}=\frac{\sqrt{K^{2} \sigma^{2}+d^{2} \mu_{s}\left(3 \mu_{c}-3 \mu_{i g}-\mu_{s}-K \sigma / d\right)}-K \sigma}{3 \mu_{c}-3 \mu_{i g}-\mu_{s}-K \sigma / d} .
$$

This means that a compact of grains of size $r>r_{m}$ can decrease its energy via grain growth with no energy barrier. A compact of grains of size $r<r_{m}$ can decrease its energy via grain shrinkage with no energy barrier. Which process actually occurs depends on the distribution of grain sizes and the relative rates of growth and shrinkage which is controlled by the temperature. The question of rates will be dealt with in the next section.

Numerical estimates for all of the parameters in Eq. (2) are shown in Table I. The volume energies can be estimated from the heat of melting of c-Si and from the heat of crystallization of a-Si as measured by DSC [3] (assuming that the compact temperature is much less than the melting and crystallization temperatures). We estimate $d$ and $\sigma$ from molecular dynamics simulations $[11,13] . \mu_{s}$ can be computed from measured values of the elastic constants for bulk $\mathrm{c}-\mathrm{Si}$ [14] and strain estimated from the density difference between c-Si and a-Si [12].

Figure 1 shows $\Delta G$ computed for a simple cubic lattice of cubic grains (chosen for simplicity of calculation, not as a realistic geometry) as a function of the cube side length $r$. In this case $K=6$, and $r_{m} \approx 10 \AA$. It is important to note that $r^{*} \approx 90 \AA$ is the smallest thermodynamically stable grain size. A compact with smaller grain size is unstable against transition to the disordered phase; however, as the only direct transformation pathway leads over the maximum at $r_{m}$, a compact with $r<r *$ is metastable against this transformation.

This free energy landscape leads to an alternate explanation for the DSC measurement that a-Si evolves $\sim 3.7 \mathrm{~kJ} / \mathrm{mol}$ of heat during low temperature annealing [3, 4], which was previously explained as the annihilation of interstitial- and vacancy-like point defects. We suggest that this is instead the heat of transformation from the PC phase to the disordered phase. From Figure 1 we see that a compact with $r=15 \AA$ would release $\sim 18 \mathrm{~kJ} / \mathrm{mol}$, which is five times larger than the observation. Some of this difference, however, is likely due to the significant uncertainties in the numerical parameters and the unrealistic geometry used. 


\section{PHASE TRANSFORMATION RATES}

We now consider the rates at which the various processes occur as a function of temperature. The relevant processes are: nucleation of the crystalline phase, growth of the crystalline phase from a pre-existing seed, and relaxation to disordered thermal equilibrium. To compute the homogeneous nucleation rate, we need to know the free energy difference associated with introducing a crystalline grain of a particular radius into the disordered matrix. Neglecting the strain contribution, we have a simple expression with surface and volume terms

$$
\Delta G_{g}(r)=K_{1} r^{3} \mu_{g}+K_{2} r^{2} \sigma,
$$

where $K_{1}, K_{2}, \sigma$, and $r$ are the same as previously, and $\mu_{g}$ is the volume free energy difference between the phases. This time, however, we wish to include the effects of temperature. Provided that the specific heats of the two phases are the same and the residual entropy of the amorphous phase is small [2], the volume free energy difference is linear in the temperature [15], and

$$
\mu_{g}=\left(\mu_{c}-\mu_{d}\right) \frac{T_{s}-T}{T_{s}},
$$

where $T$ is the temperature and $T_{s}$ is the temperature at which the phase transformation occurs [15]. In this case, $T_{s}=1000 \mathrm{~K}$, the crystallization temperature of a-Si [2].

$\Delta G_{g}(r)$ computed for a spherical grain at $T=600 \mathrm{~K}$ is shown in Figure 2(a). The critical grain size $r^{*}$ occurs at the maximum of $\Delta G_{g}$. A cluster with $r \geq r^{*}$ can decrease its energy by growing. The nucleation rate depends on the energy barrier to forming a critical-size nucleus, $\Delta \mathrm{G}_{\mathrm{g}}\left(r^{*}\right)$ via [16]

$$
I=I_{0} \exp \left(\frac{-\Delta G_{g}\left(r^{*}\right)}{k_{B} T}\right),
$$
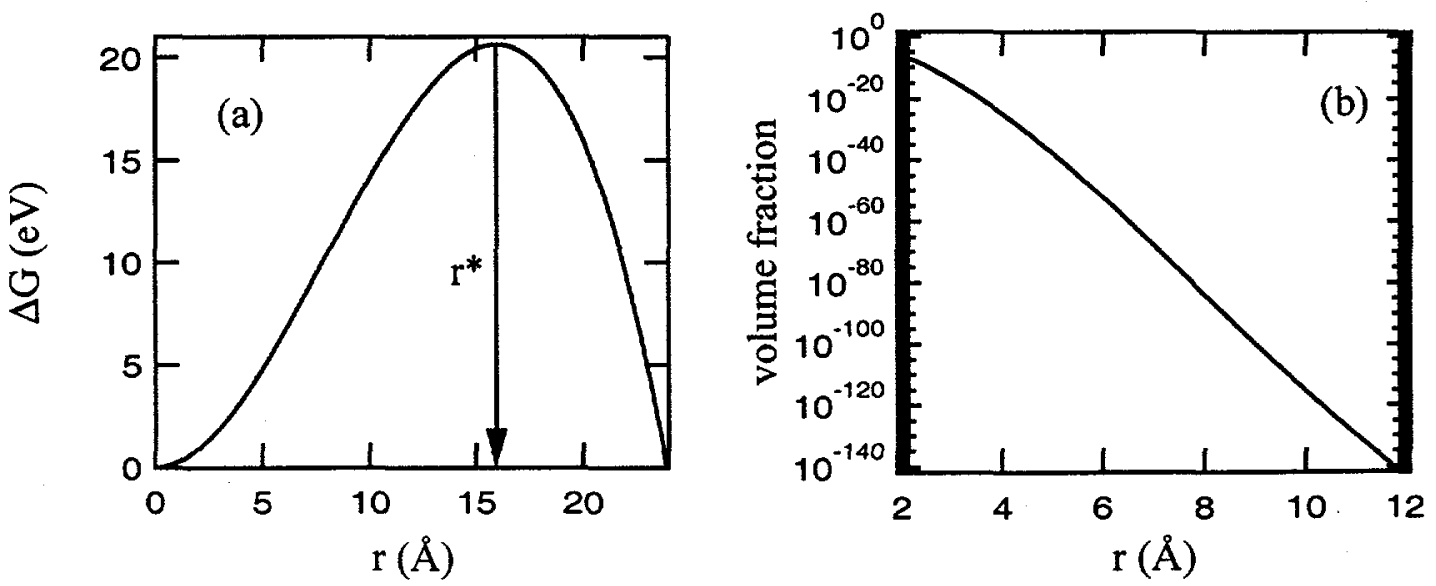

Figure 2: (a) free energy change associated with introducing a spherical crystalline embryo into the intergranular matrix as a function of the sphere radius at $T=600 \mathrm{~K}$. (b) the equilibrium volume fraction of embryos as a function of radius $T=600 \mathrm{~K}$. 
where $I_{0}$ is a constant associated with the jump frequency across the phase interface. For a liquid$\mathrm{Si} / \mathrm{c}-\mathrm{Si}$ interface and homogeneous nucleation, $I_{0}=10^{39} \mathrm{~m}^{-3} \mathrm{~s}^{-1} . I_{0}$ for the a-Si/c-Si interface is certainly smaller, but even using this value for $I_{0}$, homogeneous nucleation is negligible. $I \ll 10^{-10} \mathrm{~m}^{-3} \mathrm{~s}^{-1}$ for $T=300,600$, and $1000 \mathrm{~K}$.

We know, however, that a-Si recrystallizes at $T=1000 \mathrm{~K}$. We propose that this is due to growth of the crystal from some pre-existing seed. An estimate of the rate of this process may be made by assuming a crystalline seed and calculating the solid-phase epitaxial growth (SPEG) rate. In the fastest growth direction, $\langle 100\rangle$, the SPEG rate is given by

$$
v_{S P E G}=v_{0} \exp \left(\frac{-\Delta E^{*}}{k_{B} T}\right)
$$

where $v_{0}=4.6 \times 10^{6} \mathrm{~m} / \mathrm{s}$ and $\Delta E^{*}=2.7 \mathrm{eV}[16] . v_{S P E G}(300 \mathrm{~K})=2.1 \times 10^{-39} \mathrm{~m} / \mathrm{s}$, $v_{S P E G}(600 \mathrm{~K})=9.8 \times 10^{-17} \mathrm{~m} / \mathrm{s}$, and $v_{S P E G}(1000 \mathrm{~K})=1.1 \times 10^{-7} \mathrm{~m} / \mathrm{s}$, showing that this process only becomes significant near the observed recrystallization temperature of a-Si.

The equilibrium volume fraction distribution of grains $f(r)$ can be calculated from [15]

$$
f(r)=K_{1} r^{3} \exp \left(\frac{-\Delta G_{g}(r)}{k_{B} T}\right) .
$$

Figure 2(b) shows $f(r)$ at $T=600 \mathrm{~K}$ for $\Delta G_{g}$ shown in Figure 2(a). The very small equilibrium volume fractions (and the equilibrium value is an upper bound [15]) of the grains indicate that in equilibrium the disordered phase dominates. Therefore the observed paracrystalline structure is metastable. There is no available experimental evidence on the rate of relaxation towards the disordered state. Additional fluctuation microscopy experiments are planned to address this shortfall. However, we can argue that at intermediate temperatures the rate of relaxation should be faster than the SPEG rate from simple considerations. One contribution to the high activation energy for SPEG is that atoms from the disordered side of the a/c interface must find exactly the right spots to join the crystal [5]. For example, the disordered matrix contains five- and sevenmembered rings, while the crystal contains only six-rings. There should be greater freedom for an atom from a crystalline grain to join the disordered matrix, since it could participate in a five-, six-, or seven-membered ring. It has also been suggested [5] that coordination defects (dangling or floating bonds) at the c/a interface play a crucial role in SPEG. If that is the case, we expect the paracrystalline grains/disordered matrix interface to be particularly mobile, since in our molecular dynamics simulations of $\mathrm{PC}$ material, that boundary has a particularly high concentration of coordination defects. Both the relaxed topological constraints and the high local concentration of defects are likely to be more important at lower temperatures, which reduce the range of bond switchings atoms at the interface can explore and reduce the mobility of defects through the bulk of the film.

\section{CONCLUSIONS}

We have introduced a simple thermodynamic model of paracrystalline silicon. The model consists of a compact of strained grains which are topologically crystalline, separated by a layer of more disordered CRN-like material. This model has three regimes as a function of grain size: 
For large grains, the model is thermodynamically stable polycrystalline silicon. For intermediatesize grains, the model is metastable paracrystalline silicon. In this state, the material is unstable against transformation into a uniform disordered state, but must surmount an energy barrier to make that transition. For small grains, there is no barrier and the system transitions smoothly to the uniform disordered state.

We have also compared the rates at which homogenous crystal nucleation, recrystallization from a seed, and relaxation to the uniform disordered state take place at room temperature, under low-temperature annealing $(600 \mathrm{~K})$, and high temperature annealing $(1000 \mathrm{~K})$. Homogenous nucleation is negligible at all temperatures, while recrystallization dominates at high temperature, and we argue that relaxation to the disordered state dominates at intermediate temperatures.

\section{ACKNOWLEDGMENTS}

P.M.V. acknowledges the support of the National Science Foundation (DMR 97-03906).

\section{REFERENCES}

1. P. A. Fedders, D. A. Drabold, and S. Nakhmanson, Phys. Rev. B 58, 15624 (1998); E. Kim, and Y. H. Lee, Phys. Rev. B. 49, 1743 (1994).

2. E. P. Donovan, F. Spaepen, D. Turnbull, J. M. Poate, D. C. Jacobson, J. Appl. Phys. 57, 1795 (1985).

3. S. Roorda, S. Doorn, W. C. Sinke, P. M. L. O. Scholte, and E. van Loenen, Phys. Rev. Lett. 62, $1880(1989)$.

4. E. P. Donovan, F. Spaepen, J. M. Poate, and D. C. Jacobson, Appl. Phys. Lett. 55, 1516 (1989).

5. G. L. Olson and J. A. Roth, in Handbook of Crystal Growth Vol. 3, edited by D. T. J. Hurle (Elsevier Science, 1994), pp. 257-312, and references therein.

6. M. M. J. Treacy and J. M. Gibson, Acta Cryst. A 52, 212 (1996).

7. K. Laaziri, S Kycia, S. Roorda, M. Chicoine, J. L. Robertson, J. Wang, and S. C. Moss, Phys. Rev. Lett. 82, 3460 (1999); Phys. Rev. B 60, 13520 (1999).

8. M. M. J. Treacy, J. M. Gibson, and P. J. Keblinski, J. Non-Cryst. Solids 231, 99 (1998).

9. J. M. Gibson and M. M. J. Treacy, Phys. Rev. Lett. 78, 1074 (1997).

10. M. M. J. Treacy, P. M. Voyles, and J. M. Gibson, J. Non-Cryst. Solids 266, 150 (2000).

11 . P. Keblinski, S. R. Phillpot, D. Wolf, and H. Gleiter, Phys. Rev. Lett. 77, 2965 (1996).

12. D. L. Williamson, S. Roorda, M. Chicoine, R. Tabti, P. A. Stolk, S. Acco, and F. W. Saris, Appl. Phys. Lett. 67, 226 (1995).

13. F. Spaepen, in Amorphous Materials: Modeling of Structure and Properties, edited by V. Vitek (TMS, Warrendale, PA, 1983), p. 265.

14. W. A. Brantley, J. Appl. Phys. 44, 543 (1973).

15. W. A. Tiller, The Science of Crystallization: Microscopic Interfacial Phenomena, (Cambridge University Press, Canbridge 1991), pp. 327-382.

16. Y. Shao, F. Spaepen, and D. Turnbull, Mett. Mat. Trans. A 29, 1825 (1998). 